\title{
Stellungnahme zur geräteunterstützten cardiopulmonalen Reanimation (Device-Assisted CPR)
}

U. Klemmer, Präsident SRC

In jüngster Zeit kommen Geräte zur automatischen Thoraxkompression, z. B. LDB (Load-Distributing Band) oder LUCAS auf den Markt [1].

Die aktuell verfügbaren Literaturangaben zum Nutzen solcher Geräte sind widersprüchlich (2-4). Es bestehen Hinweise für ein schlechteres neurologisches Outcome und schlechtere Überlebensraten beim Einsatz solcher Geräte im Ver- gleich zur manuellen Thoraxkompression [2]. Diese Studie wurde deswegen vorzeitig sistiert. Zudem wurde in jüngster Zeit über schwere Komplikationen wie z.B. Aortenruptur berichtet $[5,6]$.

Der SRC empfiehlt daher derzeit, solche Geräte ausserhalb klinischer Studien weder anzuschaffen noch bei Reanimationen einzusetzen.

\section{Prise de position au sujet de la réanimation cardio-pulmonaire assistée par des appareils (Device-Assisted CPR)}

\section{U. Klemmer, président du SRC}

Des appareils de compression thoracique automatique ont récemment fait leur apparition sur le marché. On citera à titre d'exemple le LDB (Load-Distributing Band) ou le système LUCAS [1].

Les références bibliographiques actuellement disponibles contiennent des avis contradictoires quant à l'utilité de tels appareils [2-4). Selon certains indices, leur utilisation conduit à des résultats neurologiques et des taux de survie moins bons que la compression thoracique manuelle [2], raison pour laquelle l'étude citée ici a été interrompue prématurément. En outre, des rapports récents font état de complications graves telles qu'une rupture aortique $[5,6]$.

Par conséquent, le SRC recommande en ce moment de ne pas acquérir ni utiliser de tels appareils lors de réanimations en dehors d'études cliniques.

\section{Literatur/Références}

12005 American Heart Association Guidelines for CPR and ECC. Part 6: CPR Techniques and Devices. Circulatio. 2005;112(suppl ):IV-47-IV-50.

2 Hallstrom A, Rea TD, Sayre MR, Christenson J, Anton AR, Mosesso VN, et al. Manual chest compression vs use of an automated chest compression device during resuscitation following out-ofhospital cardiac arrest: a randomized trial. JAMA. 2006;295:2620-2628.

3 Ong Me, Ornato JP, Edwards DP, Dhindsa HS, Best $\mathrm{AM}$, Ines CS, et al. Use of an automated, load-distributing band chest compression device for outof-hospital cardiac arrest resuscitation. JAMA. 2006;295:2629-2637.

4 Lewis RJ, Nieman JT. Manual vs device-assisted CPR: reconciling apparently contradictory results. JAMA. 2006;295:2661-2664.

5 Englund E. Kongstad PC Active compressiondecompression CPR necessitates follow-up post mortem. Resucitation. 2006;68:161-162.

6 Englund E. Tissue injuries after CPR / abstract. Resuscitation. 2006;69(1):71-72. 